fracture, Geriatric Depression Score and treatment assignment. Women with only a new VDef but no CVFx had intermediate levels of prefracture back pain and disability. Women suffering a CVFx reported a mean of 6 days of severe back pain and 6 days of back disability for the 3 month period before the fracture, and 18 and 32 days (both $\mathrm{P}<.05$ by Wilcoxen test), respectively, for the 3 month period after the fracture. By 5-7 months postfacture mean days of severe back pain were no longer elevated $(10 ; \mathrm{P}=.44)$, but mean days of disability were still increased $(24 ; \mathrm{P}<.05)$ at $9-11$ months post fracture.

\begin{tabular}{llll}
\multicolumn{4}{l}{ Abstract SAT0179 Table 1} \\
\hline & $\begin{array}{l}\text { Severe } \\
\text { back pain } \\
(\%)\end{array}$ & $\begin{array}{l}\text { Back disability } \\
\text { score, adj. mean } \\
(\text { SE) }\end{array}$ & $\begin{array}{l}\text { Days of disability past 12 } \\
\text { months, adj. mean (SE) }\end{array}$ \\
\hline $\begin{array}{l}\text { Group 1. No new } \\
\text { CVFx or VDef }\end{array}$ & 3.9 & $0.8(0.1)$ & $13(1)$ \\
$\begin{array}{l}\text { Group 2. }>=1 \\
\text { new VDef, }\end{array}$ & 6.6 & $1.0(0.2)$ & $18(4)^{*}$ \\
$\begin{array}{l}\text { Group 3. 1 new } \\
\text { CVFx }\end{array}$ & 8.6 & $1.4(0.2)^{*}$ & $26(6)^{*}$ \\
\hline $\begin{array}{l}\text { Baseline (prefracture) back pain and disability }\left({ }^{*} \mathrm{P}<.05 \text { vs Group 1). }\right.\end{array}$
\end{tabular}

Conclusion Older women who have chronic back pain are more likely to suffer a CVFx. In those who have a CVFx, average back disability remains above prefracture levels for at least a year after the fracture. Prevention of fractures may be especially beneficial in older women with chronic back pain.

\section{SAT0180 DO REHABILITATION FACILITIES DIAGNOSE AND TREAT OSTEOPOROSIS IN HIP FRACTURE PATIENTS BETTER THAN ACUTE CARE HOSPITALS?}

${ }^{1} \mathrm{AG} \mathrm{Juby,}{ }^{2} \mathrm{CM}$ De Geus-Wenceslau. ${ }^{1}$ Geriatric Medicine, University of Alberta, Edmonton, Canada; ${ }^{2}$ Geriatric Medicine and Rheumatology, Curitiba, Brazil

\subsection{6/annrheumdis-2001.667}

Background One in four women and one in eight men over 50 years of age will suffer from osteoporosis. The lifetime prevalence of hip fracture is $18 \%$ for women, $6 \%$ for men. Mortality within a year of hip fracture is $6-44 \%$. Morbidity is also significant, with many not returning to their premorbid level of function. Proven efficacious pharmacologic therapies are available to treat osteoporosis.

Objectives The objective of this study was to evaluate the diagnosis and treatment of osteoporosis in seniors after hip fracture both in the acute care hospital and in the rehabilitation facility, and to see if they differ.

Methods A retrospective chart review on consecutive hip fracture patients (over $65 \mathrm{yrs}$ ) admitted to an acute tertiary care referral hospital was carried out. Osteoporosis diagnosis and treatment was recorded. Additional data was collected on osteoporosis investigations including bone densitometry. The patients were then followed up in the rehabilitation facility and the same data collected. The results from the two groups were then compared.

Results Data was collected on 311 patients in acute care. Average age was 86 years, average length of stay in acute care was 11 days. 18 died and 41 either went straight home or to long term care. Data was unavailable in 26 patients. Therefore 226 were followed up in the rehabilitation facility. Osteoporosis was diagnosed on admission to: acute care in $11.9 \%$; rehabilitation in $9.7 \%$ and on discharge from: acute care in 15.4\%; and from rehabilitation in 11.2\%. Osteoporosis was treated (any treatment) in acute care hospitals in $13 \%$ on admission and $9.7 \%$ on discharge and in rehabilitation facilities $12.8 \%$ and $10.2 \%$ respectively. In the majority of cases the treatment was calcium alone, specific osteoporotic drugs were used in $<4 \%$ in both groups. There was no statistically significant differences between the groups, apart from the use of Vitamin D which was significantly less $(p<0.05)$ in the rehabilitation group compared to the acute care group.

Conclusion Rehabilitation facilities are no better than acute care hospitals at diagnosing and pharmacologically treating osteoporosis in seniors after hip fracture. The rate of treatment in both groups is unacceptably low. In spite of their more holistic and multidisciplinary focus the rehabilitation facilities still fared poorly in their approach to this disease. This puts the onus on primary care physicians to provide adequate osteoporosis treatment in this group of patients, otherwise elderly people will continue to be under-managed for this disease.

\section{SAT0181 DOES A REVERSE YENTL SYNDROME EXIST IN OSTEOPOROSIS?}

${ }^{1}$ SE Green, ${ }^{2}$ SJ Davies, 'EJ Price, 'DA Collins. 'Rheumatology, Princess Margaret Hospital, Swindon; ' 2 Pharmacology and Therapeutics, Royal Hallamshire Hospital, Sheffield, UK

\subsection{6/annrheumdis-2001.668}

Background The Yentl syndrome ${ }^{1}$ occurs where a woman has to masquerade as a man in order to receive the same treatment. It was described in the management of ischaemic heart disease where women were shown to be undertreated in comparison to men until they suffered a defining event such as myocardial infarction.

Low energy fractures are a manifestation of established and developing osteoporosis. Women who sustain a wrist fracture within ten years of the menopause are at an eight-fold increased risk of hip fracture compared to the general population. ${ }^{2}$ Osteoporosis also carries an appreciable risk of hip fracture in men. ${ }^{3}$

Objectives We hypothesised that men may receive less intervention than women after low energy fractures.

Methods All patients 40 years of age and over who had a distal radius fracture in a 3 month period (from 1.12.1999) at a General Hospital were identified from radiograph reports. The manner of injury and subsequent management were assessed from self-completed patient questionnaires. Low energy fractures were defined as a fall from standing height or less, at walking speed or slower and confirmed where necessary by case note review or telephone. Men and women who sustained low energy fractures were compared for advice, investigation and treatment relating to osteoporosis.

Results Ninety-one of 105 people identified (87\%) returned the questionnaire, of whom $72(79 \%)$ were judged to have sustained low energy fractures $[12$ males (17\%) and 60 females $(83 \%)$, with average age at fracture 63 (range 43?85) and 68 years (range 40?92) respectively]. By questionnaire, 8 women (15\%) but none of the men were advised at the time of the fracture of the possibility of osteoporosis. Nine women (16\%) and none of the men had been investigated ( 8 cases by DEXA scan, and one case by bone ultrasound). Sixteen women (28\%) were currently taking bone protection therapy (5 hormone replacement therapy, 
3 etidronate, 2 calcium and vitamin D supplements and 5 calcium supplements alone). None of the men were taking any medication for osteoporosis, a difference in comparison to women of borderline significance $(\mathrm{p}=0.055$, Fisher?s Exact Test).

Conclusion Only a small minority of patients with low energy fractures were made aware of the possibility of having osteoporosis and were adequately investigated or treated. Men accounted for $17 \%$ of presentations, but the complete absence of advice and appropriate investigation and treatment of osteoporosis among males is striking. There is much scope for improvement in the management of low energy fractures, especially in men. A reverse of the Yentl syndrome may exist in osteoporosis.

\section{REFERENCES}

1 Healy B. The Yentl syndrome. N Engl J Med. 1991:325:274-6

2 Wigderowitz CA, Rowley DI, Mole PA, et al. Bone mineral density of the radius in patients with Colles? fracture. Br J Bone Joint Surg. 2000;82B:87-9

3 Nyquist F, Gardsell P, Sernbo I, et al. Assessment of sex hormones and bone mineral density in relation to occurrence of fracture in men. Bone 1998;22:147-51

\section{SAT0182 ASSESSMENT OF BONE METABOLISM IN PATIENTS WITH CHRONIC CONNECTIVE TISSUE DISEASES}

I Korczowska, P Leszczynski, P Hrycaj, JK Lacki. Department of Rheumatology and Clinical Immunology, University School of Medicine, Poznan, Poland

\subsection{6/annrheumdis-2001.669}

\section{Background}

Objectives The aim of our recent study was to evaluate bone metabolism in rheumatoid arthritis (RA) and systemic lupus erythematosus patients (SLE).

Methods 306 women (90 RA, 38 SLE patients and 178 healthy volunteers) were involved in the study. All SLE and 44 RA patients were on chronic glucocorticosteroids theraphy (GCS). Average age was: $46.4 \pm 12.8$ yrs, $52.0 \pm 12.0$ yrs in SLE group and the control (respectively). Disease duration and duration of GCS therapy in SLE patients was: $108 \pm 82$ months. Total dose of GCS was the highest in SLE pts $(25.3 \pm 28.19 \mathrm{~g})$. Patients with RA were divided into two groups: treated with GCS (average age: $52.5 \pm 12.4 \mathrm{yrs}$, disease duration and duration of GCS therapy: $122 \pm 102$ months, total dose of GCS: $7.4 \pm 8.3 \mathrm{~g}$ ): and who were not treated with GCS (average age: $54.3 \pm 9.7$ yrs, disease duration: $134 \pm 120$ months). In all patients the following markers of bone metabolism were measured: osteocalcin, procolagen $\mathrm{C}$, alkaline phosphatase-bone fraction, carboxyterminal telopeptides of type I collagen (CTX), procollagen type I carboxyterminal propeptide (PICP), deoxypyridynoline. The disease activity was assessed by measuring serum levels of proinflammatory cytokines (IL-1a, IL-6, TNF-alpha, GM-CSF) and acute phase markers (ESR, CRP, AGP, AGP-RC, ACT).

Results Concentration of biochemical markers of osteoporosis suggesting increased bone metabolism in RA patients indicates significantly higher bone loss in this group than in SLE patients in spite of the highest dosage of GCS. There was no significantly differences between bone turnover in RA patients treated and not treated with GCS.

Conclusion The serum levels of proinflammatory cytokines and acute phase markers confirm the highest stage of the disease activity in RA patients.
SAT0183 BONE MINERAL DENSITY IN WOMEN WITH RHEUMATOID ARTHRITIS

AN Dimic, V Radenkovic, S Milenkovic, Z Markovic, A Stankovic. The Institute for Prevention, Treatment and Rehabilitation of Rheumatic and Cardiovascular Diseases, Niska Banja Institue, Niska Banja, Yugoslavia

\subsection{6/annrheumdis-2001.670}

Background Rheumatoid arthritis (RA) has a significant influence on bone loss in both periph-eral (juxtaarticular osteoporosis) and axial skeleton. Glucocorticoids (GC) are often used in the therapy of RA and contribute significantly to favourable disease outcome but have been accused to cause a bone loss in these patients.

Objectives To assess the influence of GC on the occurrence of osteoporosis in RA patients.

Methods Hundred and seven women with RA were evaluated. They were divided in two age-groups: 40-49 and 50-59. The assessment of bone mineral density was performed by dual energy $\mathrm{x}$-ray absorptiometry (DEXA), using Lunar DPX densitometer. Anterior-posterior spine scan of lumbar vertebrae L1-L4 was performed.

Results The following average values of bone mineral density (BMD) were found: $1.116 \mathrm{~g} / \mathrm{cm}^{2}$ for age-group of $40-49$ and $0.942 \mathrm{~g} / \mathrm{cm}^{2}$ for $50-59$ years old patients. In comparison with control group (300 healthy women of the same age-groups) we found significantly lower BMD in RA patients $(\mathrm{p}<0.05$ for the age group of 40-49 and $\mathrm{p}<0.001$ for age-group of 50-59).

Annual rate of bone loss in both control and group of RA patients was also evaluated. In the control group annual loss was $0.57 \%$, and in group of RA patients $1.45 \%$ ( $\mathrm{p}<0.001$ ).

In RA patients treated with GC significantly lower BMD in comparison with RA patients not taking these drug was found.

\begin{tabular}{clll}
\multicolumn{2}{l}{ Abstract SAT0183 Table 1 } & & \\
\hline Age & $\begin{array}{l}\text { RA without GC } \\
\text { g/cm }\end{array}$ & $\begin{array}{l}\text { RA with GC } \\
\text { g/cm }\end{array}$ & p \\
\hline 40-49 years & $1.129 \pm 0.104$ & $1.066 \pm 0.104$ & $<0.05$ \\
$50-59$ years & $0.965 \pm 0.136$ & $0.897 \pm 0.112$ & $<0.05$ \\
\hline
\end{tabular}

Conclusion Osteopenia defined as BMD below one standard deviation from the corresponding young adult normal value was found in 26\% of RA patients aged 40-49 and in 45\% RA patients aged 50-59.

In conclusion this study has confirmed that the disease itself, therapy with glucocorticoids and oestrogen deficiency during menopause influence bone loss in RA patients.

\section{Diagnostics and imaging procedures}

\section{AB0193 ELECTROMYOGRAPHY AND NERVE CONDUCTION IN PATIENTS ON REGULAR HAEMODIALYSIS}

AF El Ghobarey, W Kamel, E Abdel Mobdy. Internal Medicine, Al Azhar University, Cairo, Egypt

10.1136/annrheumdis-2001.671

Background 